\title{
Contemporary Mathematicians
}

\author{
Gian-Carlo Rota
}

Editor 


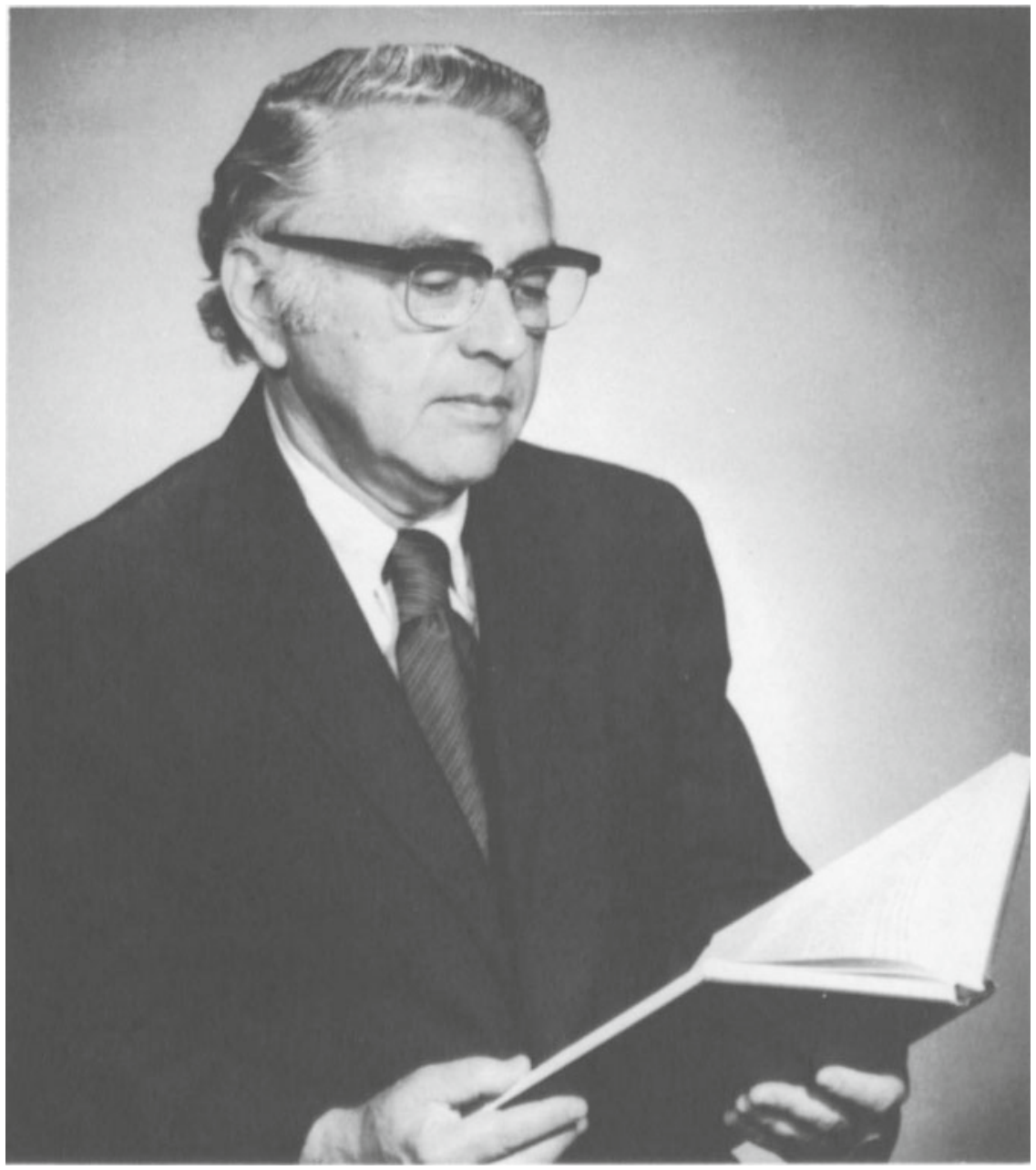

Nathan Jacobson, 1972 


\title{
Nathan Jacobson \\ Collected Mathematical Papers \\ Volume 2 \\ (1947-1965)
}

\author{
Birkhäuser
}

Boston - Basel - Berlin

1989 


\title{
Nathan Jacobson \\ Department of Mathematics \\ Yale University \\ New Haven, CT 06520
}

\author{
Library of Congress Cataloging-in-Publication Data \\ Jacobson, Nathan, 1910- \\ [Selections. 1989] \\ Collected mathematical papers / Nathan Jacobson. \\ p. cm.-(Contemporary mathematicians) \\ Includes bibliographies.
}

ISBN-13: 978-1-4612-8215-0

e-ISBN-13: 978-1-4612-3694-8

DOI: $10.1007 / 978-1-4612-3694-8$

1. Mathematics. I. Title. II. Series. QA3.J3325 1989

$510-\mathrm{dc} 20$

89-14889

CIP

All rights reserved. No part of this publication may be reproduced, stored in a retrieval system, or transmitted, in any form or by any means, electronic, mechanical, photocopying, recording or otherwise, without prior permission of the copyright owner.

(C) Birkhäuser Boston, 1989

Softcover reprint of the hardcover 1st edition 1989 


\section{Bibliography of Nathan Jacobson's Books and Papers}

Note: Boldface numbers at the end of each entry denote the volume in which the entry appears.

Books

The Theory of rings: Mathematical Surveys, No. II, Amer. Math. Soc., 1943(Russian Translation, 1947).

Lectures in Abstract Algebra: Vol. 1, Basic Concepts, D. Van Nostrand Co. Inc., 1951 (SpringerVerlag reprint, 1975; Chinese translation, 1966).

Lectures in Abstract Algebra: Vol. 2, Linear Algebra, D. Van Nostrand Co. Inc., 1953 (SpringerVerlag reprint, 1975; Chinese translation, 1960).

Lectures in Abstract Algebra: Vol. 3, Theory of Fields and Galois Theory, D. Van Nostrand Co. Inc., 1964 (Springer-Verlag reprint, 1975).

Structure of Rings, Amer. Math. Soc. Colloquium Publications, Vol. 37, 1956, 1964 (Russian translation, 1961).

Lie Algebras, Interscience Publishers (John Wiley and Sons), 1962 Interscience Tracts in Pure and Applied Mathematics, No. 10 (Dover reprint, 1979; Russian translation, 1964; Chinese translation, 1964).

Structure and Representations of Jordan Algebras, Amer. Math. Soc. Colloquium Publications, Vol. 39, 1968.

Lectures on Quadratic Jordan Algebras, Tata Institute of Fundamental Research, Bombay, 1969.

Exceptional Lie Algebras, Lecture Notes in Pure and Applied Mathematics, Marcel Dekker Inc., New York, 1971.

Basic Algebra I, W. H. Freeman and Co., New York, 1974; second edition, 1985.

Pi-Algebras: An Introduction, Springer Verlag, 1975.

Basic Algebra II, W. H. Freeman and Co., New York, 1980; second edition, 1989.

Structure Theory of Jordan Algebras, University of Arkansas Lecture Notes in Mathematics, 1981.

Finite Dimensional Division Algebras (with David Saltman), Springer-Verlag Grundlehre Series, in press.

\section{Papers}

[1] "Non-commutative polynomials and cyclic algebras", (Princeton University dissertation) Annals of Math. 35 (1934) 197-208. (1)

[2] "A note on non-commutative polynomials", Annals of Math. 35 (1934) 209-210. (1)

[3] "Locally compact rings" (with O. Taussky), Proc. Nat. Acad. Sci. 21 (1935) 106-108. (1)

[4] "Rational methods in the theory of Lie algebras", Annals of Math. 36 (1935) 875-881. (1)

[5] "On pseudo-linear transformations", Proc. Nat. Acad. Sci. 21 (1935) 667-670. (1)

[6] "Totally disconnected locally compact rings", Amer. J. Math. 58 (1936) 433-449. (1)

[7] "Simple Lie algebras of type A", Proc. Nat. Acad. Sci. 23 (1937) 240-242. (1)

[8] "Pseudo-linear transformations", Annals of Math. 38 (1937) 484-507. (1)

[9] "A class of normal simple Lie algebras of characteristic zero", Annals of Math. 38 (1937) 508-517. (1) 
[10] “A note on non-associative algebras", Duke Math. J. 3 (1937) 544-548. (1)

[11] “Abstract derivation and Lie algebras", Trans. Amer. Math. Soc. 42 (1937) 206-224. (1)

[12] "p-Algebras of exponent p", Bull. Amer. Math. Soc. 43 (1937) 667-670. (1)

[13] "A note on topological fields", Amer. J. Math. 59 (1937) 889-894. (1)

[14] "Simple Lie algebras of type A", Annals of Math. 39 (1938) 181-188. (1)

[15] "Simple Lie algebras over a field of characteristic zero", Duke Math.J. 4(1938) 534-551. (1)

[16] "Normal semi-linear transformations", Amer. J. Math. 61 (1939) 45-58. (1)

[17] "An application of E. H. Moore's determinant of a Hermitian matrix", Bull. Amer. Math. Soc. 45 (1939) 745-848. (1)

[18] "Structure and automorphisms of semi-simple Lie groups in the large", Annals of Math. 40 (1939) 755-763. (1)

[19] "Cayley numbers and normal simple Lie algebras of type G", Duke Math. J. 5 (1939) 775-783. (1)

[20] "The fundamental theorem of the Galois theory for quasi-fields", Annals of Math. 41 (1940) 1-7. (1).

[21] "A note on hermitian forms", Bull. Amer. Math. Soc. 46 (1940) 264-268. (1)

[22] "Restricted Lie algebras of characteristic p", Trans. Amer. Math. Soc. 50 (1941) 15-25. (1)

[23] "Classes of restricted Lie algebras of characteristic $p$ I", Amer. J. Math. 63 (1941) 481-515. (1)

[24] "Classes of restricted Lie algebras of characteristic p II", Duke Math. J. 10 (1943) 107-121. (1)

[25] "An extension of Galois theory to non-normal and non-separable fields", Amer. J. Math. 66 (1944) 1-29. (1)

[26] "Schur's theorems on commutative matrices", Bull. Amer. Math. Soc. 50 (1944) 431-436. (1)

[27] "Relations between the composites of a field and those of a subfield", Amer. J. Math. 66 (1944) 636-644. (1)

[28] "Galois theory of purely inseparable fields of exponent one", Amer. J. Math. 66 (1944) 645-648. (1)

[29] "Construction of central simple associative algebras", Annals of Math. 45 (1944) 658-666. (1)

[30] "The equation $x^{\prime}=x d-d x=b "$, Bull. Amer. Math. Soc. 50 (1944) 902-905. (1)

[31] "Structure theory of simple rings without finiteness assumptions", Trans. Amer. Math. Soc. 57 (1945) 228-245. (1)

[32] "The radical and semi-simplicity for arbitrary rings", Amer. J. Math. 67 (1945) 300-320. (1)

[33] "Structure theory for algebraic algebras of bounded degree", Annals of Math. 46 (1945) 695-707. (1)

[34] "A topology for the set of primitive ideals in an arbitrary ring", Proc. Nat. Acad. Sci. 31 (1945) 333-338. (1)

[35] "On the theory of primitive rings", Annals of Math. 48 (1947) 8-21. (1)

[36] "A note on division rings", Amer. J. Math. 69 (1947) 27-36. (1)

[37] "Isomorphisms of Jordan rings", Amer. J. Math. 70 (1948) 317-326. (1)

[38] "The center of a Jordan ring", Bull. Amer. Math. Soc. 54 (1948) 316-322. (1)

[39] "Lie and Jordan triple systems", Amer. J. Math. 71 (1949) 149-170. (2)

[40] "Classification and representation of semi-simple Jordan algebras" (with F. D. Jacobson), Trans. Amer. Math. Soc. 65 (1949) 141-169. (2)

[41] "Derivation algebras and multiplication algebras of semi-simple Jordan algebras", Annals of Math. 50 (1949) 866-874. (2)

[42] "Enveloping algebras of semi-simple Lie algebras", Can. J. Math. 2 (1950) 257-266. (2)

[43] "Some remarks on one-sided inverses", Proc. Amer. Math. Soc. 1 (1950) 352-355. (2)

[44] "Jordan homomorphisms of rings" (with C. E. Rickart), Trans. Amer. Math. Soc. 69 (1950) 479-502. (2) 
[45] "Completely reducible Lie algebras of linear transformations", Proc. Amer. Math. Soc. 2 (1951) 105-113. (2)

[46] "General representation theory of Jordan algebras", Trans. Amer. Math. Soc. 70 (1951) 509-530. (2)

[47] "Une généralization du théorème d'Engel", C. R. Acad. Sci. 234 (1952) 679-681. (2)

[48] "Homomorphisms of Jordan rings of self-adjoint elements" (with C. E. Rickart), Trans. Amer. Math. Soc. 72 (1952) 310-322. (2)

[49] "A note on Lie algebras of characteristic p", Amer. J. Math. 74 (1952) 357-359. (2)

[50] "Operator commutativity in Jordan algebras", Proc. Amer. Math. Soc. 3 (1952) 973-976. (2)

[51] "A Kronecker factorization theorem for Cayley algebras and the exceptional simple Jordan algebra", Amer. J. Math. 76 (1954) 447-452. (2)

[52] "Structure of alternative and Jordan bimodules", Osaka Math. J. 6 (1954) 1-71. (2)

[53] "A note on automorphisms and derivations of Lie algebras", Proc. Amer. Math. Soc. 6 (1955) 281-283. (2)

[54] "Commutative restricted Lie algebras", Proc. Amer. Math. Soc. 6 (1955) 476-481. (2)

[55] “A note on two dimensional division ring extensions", Amer. J. Math. 77 (1955) 593-599. (2)

[56] "A theorem on the structure of Jordan algebras", Proc. Nat. Acad. Sci. 42 (1956) 140-147. (2)

[57] "Generation of separable and central simple algebras", J. Math. Pures Appl. 36 (1957) 217-227. (2)

[58] "On reduced exceptional simple Jordan algebras" (with A. A. Albert), Annals of Math. 66 (1957) 400-417. (2)

[59] "On Jordan algebras with two generators" (with L. J. Paige), J. Rat. Mech. Anal. 6 (1957) 895-906. (2)

[60] "Composition algebras and their automorphisms", Rend. Circ. Math. Palermo 7 (Series II) (1958) 1-26. (2)

[61] "Nilpotent elements in semi-simple Jordan algebras", Math. Ann. 136 (1958) 375-386. (2)

[62] "A note on three dimensional simple Lie algebras", J. Math. Mech. 7 (1958) 823-832. (2)

[63] "Some groups of transformations defined by Jordan algebras I", J. Reine Angew. Math. 201 (1959) 178-195. (2)

[64] "Some groups of transformations defined by Jordan algebras II", J. Reine Angew. Math. 204 (1960) 74-98. (2)

[65] "Some groups of transformations defined by Jordan algebras III", J. Reine Angew. Math. 207 (1961) 61-85. (2)

[66] "Macdonald's theorem on Jordan algebras", Archiv Math. 13 (1962) 241-250. (2)

[67] "A coordinatization theorem for Jordan algebras", Proc. Nat. Acad. Sci. 48 (1962) 11541160. (2)

[68] “A note on automorphisms of Lie algebras", Pac. J. Math. 12 (1962) 303-315. (2)

[69] "Generic norm of an algebra", Osaka Math. J. 15 (1963) 25-50. (2)

[70] "Clifford algebras for algebras with involution of type D", J. Algebra 1 (1964) 288-300. (2)

[71] "Triality and Lie algebras of type $\mathrm{D}_{4}$ ", Rend. Circ. Math. Palermo 13 (Series II) (1964) $1-25 .(2)$

[72] "Cartan subalgebras of Jordan algebras", Nagoya Math. J. 27 (1966) 591-609. (3)

[73] "Structure theory for a class of Jordan algebras", Proc. Nat. Acad. Sci. 55 (1966) 243-251. (3)

[74] "Quadratic Jordan algebras of quadratic forms with base points" (with K. McCrimmon), Indian Math. Soc. 35 (1971) 1-45. (3)

[75] "Generally algebraic quadratic Jordan algebras" (with J. Katz), Scri. Math. 29 (1971) 215-227. (3) 
[76] "Structure groups and Lie algebras of Jordan algebras of symmetric elements of associative algebras with involution", Adv. Math. 20 (1976) 106-150. (3)

[77] "Localization of Jordan algebras" (with K. McCrimmon and M. Parvathi), Commun. Algebra 6 (9) (1978) 911-958. (3)

[78] "Bimodule structure of certain Jordan algebras relative to subalgebras with one generator", Hokkaido Math. J. 10 (1981) 333-342. (3)

[79] "Some application of Jordan norms to associative algebras", Adv. Math. 48 (1983) 149165. (3)

[80] "Forms of the generic norm of a separable algebra", J. Algebra 86 (1984) 76-84. (3)

[81] "Some projective varieties defined by Jordan algebras", J. Algebra 97 (1985) 565-598. (3)

[82] "Jordan algebras of real symmetric matrices", Algebras, Groups and Geometries, 4 (1987) 291-304. (3)

\section{Expository Papers}

[83] "The Classical Groups by Hermann Weyl" (A book review), Bull. Amer. Math. Soc. 46 (1940) 592-595. (3)

[84] "Representation theory for Jordan rings", Proc. Intl. Congr. Math. (1950) 2 37-43. (3)

[85] "Le problème de Kurosch", Séminaire Bourbaki 64 (1951-1952) 295-303. (3)

[86] "Some aspects of the theory of representations of Jordan algebras", Proc. Intl. Congr. Math. (1954) 3 28-33. (3)

[87] "Jordan algebras", Report of a Conference on Linear Algebras, Nat. Acad. Sci-Nat. Research Council 502 (1957) 12-19. (3)

[88] "Representation theory of Jordan algebras, Some Aspects of Ring Theory", Cen. Int. Mat. Est. 1966. (3)

[89] "Forms of algebras; Some Recent Advances in Basic Sciences", Academic Press (1966) 41-71. (3)

[90] "Associative algebras with involution and Jordan algebras", Proc. K. Akad. van Wetenschappen 69 (1966) 202-212. (3)

[91] "Connections between associative and Jordan rings", Ist. Naz. Alta Math. Symp. Math. 9 (1972) 261-268. (3)

[92] "Abraham Adrian Albert" (an obituary), Bull. Amer. Math. Soc. 80 (1974) 1076-1093. (3)

[93] "PI-Algebras, Ring Theory", Proceedings of Oklahoma Conference, edited by B. R. MacDonald, Marcel Dekker (1974) 1-30. (3)

[94] "Some recent developments in the theory of PI-algebras", Proceedings Winter School, Reinhardsbrunn, German Democratic Republic (1976) 17-21. (3)

[95] "Some recent developments in the theory of algebras with polynomial identities, I. Razmyslov's central polynomial, II. The Artin-Procesi theorem, III. On Shirshov's local finiteness theorems", Proceedings 18th SRI Springer-Verlag Canberra, 1978, 8-46. (3)

[96] "Survey of Jordan structure theory", Southeast Asian Bull. Math. 5 (1981) 27-38. (3)

[97] Emmy Noether Collected Papers (Introduction), Springer-Verlag, 1983, 16-26. (3)

[98] "Brauer factor sets, Noether factor sets and crossed products, Emmy Noether in Bryn Mawr, edited by Srinivasan and Sally, Springer-Verlag, 1983, 1-20. (3)

\section{Papers Listed by Topic}

(Ore) Skew Polynomial Domains: 1, 2, 5, 8, 16.

Topological Algebra: 3, 6, 13. 
Lie Algebras and General Non-associative Algebras: 4, 7, 9, 10, 11, 14, 15, 19, 22, 23, 24, 39, 42 , $45,47,49,53,54,62,68,69,71$.

Galois Theory: 20, 25, 27, 28, 29, 36, 55 .

General Structure Theory of Rings: $31,32,33,34,35,43,55,93,94,95$.

Jordan Rings: $\quad 37,38,39,40,41,44,46,48,50,51,61,63,64,65,66,67,69,72,73,74,75,76$, $77,78,79,80,81,82,84,86,87,88,90,91,96$.

Associative Algebras: 1, 12, 29, 57, 70, 79, 85, 98.

Miscellaneous: $\quad 17,18,21,26,30,60,92,97$. 


\title{
Doctoral Students \\ of Nathan Jacobson
}

\author{
University of North Carolina
}

Charles L. Carroll, Jr. Normal simple Lie algebras of type D and order 28 over a field of characteristic zero (1945).

Yale University

Eugene Schenkman A theory of subinvariant Lie algebras (1950).

Charles $W$. Curtis Additive ideal theory in general rings (1951).

William G. Lister A structure theory of Lie triple systems (1951).

Henry G. Jacob A theorem on Kronecker products (1953).

George B. Seligman Lie algebras of prime characteristic (1954).

Morris Weisfeld Derivations in division rings (1954).

Bruno Harris Galois theory of Jordan algebras (1956).

Earl J. Taft Invariant Wedderburn factors (1956).

Dallas W. Sasser On Jordan matrix algebras (1957).

Maria J. Woneburger On the group of similitudes and its projective group (1957).

Tae-II Suh On isomorphisms of little projective groups of Cayley planes (1961).

Herbert F. Kreimer, Jr. Differential, difference, and related operational rings (1962).

Charles M. Glennie Identities in Jordan algebras (1963).

David A. Smith On Chevalley's method in the theory of Lie algebras and linear groups of prime characteristic (1963).

Dominic C. Soda Groups of type $\mathrm{D}_{4}$ defined by Jordan algebras (1964).

Harry P. Allen Jordan algebras and Lie algebras of type $\mathrm{D}_{4}(1965)$.

Eugene A. Klotz Isomorphisms of simple Lie rings (1965).

Kevin M. McCrimmon Norms and noncommutative Jordan algebras (1965).

Joseph C. Ferrar On Lie algebras of type $\mathrm{E}_{6}(1966)$.

Daya-Nand Verma Structure of certain induced representations of complex semi-simple Lie algebras (1966).

Lynn Barnes Small Mapping theorems in simple rings with involution (1967).

John R. Faulkner Octonion planes defined by quadratic Jordan algebras (1969).

Samuel R. Gordon On the automorphism group of a semi-simple Jordan algebra of characteristic zero (1969).

Michel Racine The Arithmetics of quadratic Jordan algebras (1971).

Jerome M. Katz Automorphisms of the lattice of inner ideals of certain quadratic Jordan algebras (1972).

Ronald Infante Strongly normal difference extensions (1973).

Louis H. Rowen On algebras with polynomial identity (1973).

Georgia M. Benkart Inner ideals and the structure of Lie algebras (1974).

David J. Saltman Azumaya algebras over rings of characteristic $p$ (1976).

Robert A. Bix Separable Jordan algebras over commutative rings (1977).

Leslie Hogben Radical classes of Jordan algebras (1978).

Craig L. Huneke Determinantal ideals and questions related to factoriality (1978). 


\section{Chronology}

September 8, 1910

\section{0}

1934

1934-1935

1935-1936

1936-1937

1937-1938

1938-1940

1940-1941

1941-1942

1942-1943

1943-1947

1947-1949

1949-1961

1961-1963

1963-1981

$1981-$

Summer, 1947

1951-1952

1957-1958

Oct. 1964-Jan. 1965

Spring, 1965

Spring, 1969

Oct. 1981-Jan. 1982

Sept.-Nov. 1983

Nov.-Dec. 1983

Feb.-March 1984

Sept.-Oct. 1985

April 1988

Visiting Lecturer

1951-1952

1954
Born, Warsaw, Poland (U.S. Citizen)

A.B. University of Alabama

Ph.D. Princeton University

Assistant, Institute for Advanced Study

Lecturer, Bryn Mawr College

National Research Council Fellow, University

of Chicago

Instructor, University of North Carolina

Assistant Professor, University of North Carolina

Visiting Associate Professor, Johns Hopkins

University

Associate Professor, University of North Carolina

Associate Ground School Instructor, University of North Carolina

Associate Professor, Johns Hopkins University

Associate Professor, Yale University

Professor, Yale University

James E. English Professor, Yale University

Henry Ford II Professor, Yale University

Henry Ford II Professor Emeritus, Yale University

Visiting Professor, University of Chicago

Fulbright Scholar, University of Paris

Visiting Professor, University of Paris

Visiting Professor, University of Chicago

Lecturer, Mathematical Society of Japan

Visiting Professor, Tata Institute of Fundamental

Research

Visiting Professor, ETH, Zurich

Visiting Professor, Nanjing University, People's

Republic of China

Visiting Professor, Taiwan National University,

Republic of China

Visitor, Center for Advanced Studies, University of Virginia

Visiting Professor, Pennsylvania State University

John Hasbrouck Van Vleck Distinguished Visiting Professor, Wesleyan University

Australia, Israel, Italy, People's Republic of China, University of Texas

Guggenheim Fellow

Elected Member of National Academy of Sciences 
1960

1971-1973

1972

1972

1972-1974

1981
Elected Member of American Academy of Arts and Sciences

President of American Mathematical Society Honorary D.Sc., University of Chicago

Honorary Member of London Mathematical Society

Vice President of International Mathematical Union

Sesquicentennial Honorary Professor, University of Alabama 


\section{Preface}

This collection contains all my published papers, both research and expository, that were published from 1934 to 1988 . The research papers arranged in chronological order appear in Volume I and II and in the first part of Volume III. The expository papers, which are mainly reports presented at conferences, appear in chronological order in the last part of Volume III. Volume I covers the period 1910 to 1947, the year I moved to Yale, Volume II covers the period 1947 to 1965 when I became Chairman of the Department at Yale and Volume III covers the period from 1965 to 1989, which goes beyond my assumption of an emeritus status in 1981. I have divided the time interval covered in each volume into subintervals preceded by an account of my personal history during this period, and a commentary on the research papers published in the period. I have omitted commentaries on the expository papers and have sorted out the commentaries on the research papers according to the principal fields of my research.

The personal history has been based on my recollections, checked against written documentation in my file of letters as well as diaries. One of these was a diary I kept of my trip to the USSR in 1961; the others were diaries Florie (Florence) kept during other major visits abroad. I have also consulted Professor A. W. Tucker on historical details on Princeton during the 1930's.

The material on personal history and commentary has had critical readings by Florie, by George Seligman and by Kevin McCrimmon. I take this opportunity to express my sincere thanks to them for numerous suggestions which have resulted in an improved manuscript.

Nathan Jacobson

Hamden, Connecticut

September 25, 1989 


\section{Table of Contents}

Bibliography of Nathan Jacobson's Books and Papers $\ldots \ldots \ldots \ldots \ldots \ldots . \quad \mathbf{v}$

Doctoral Students of Nathan Jacobson $\ldots \ldots \ldots \ldots \ldots \ldots \ldots \ldots \ldots \ldots \ldots \ldots \ldots \ldots$

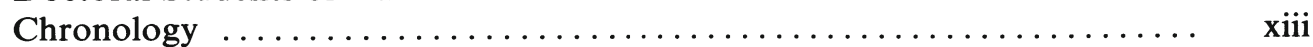

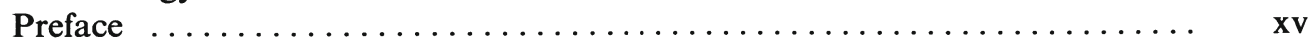

Personal History and Commentary $(1947-1955) \ldots \ldots \ldots \ldots \ldots \ldots \ldots \ldots$

Research Papers, 1947-1955

[39] Lie and Jordan triple systems ........................ 17

[40] Classification and representation of semi-simple Jordan algebras

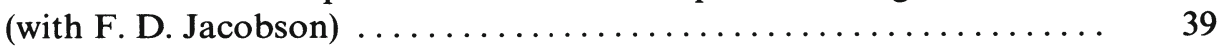

[41] Derivation algebras and multiplication algebras of semi-simple

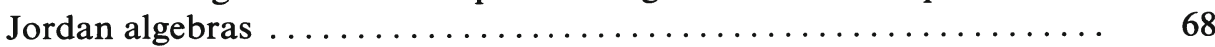

[42] Enveloping algebras of semi-simple Lie algebras $\ldots \ldots \ldots \ldots \ldots \ldots \ldots$

[43] Some remarks on one-sided inverses $\ldots \ldots \ldots \ldots \ldots \ldots \ldots \ldots \ldots . \ldots \ldots$

[44] Jordan homomorphisms of rings (with C. E. Rickart) . . . . . . . . 93

[45] Completely reducible Lie algebras of linear transformations . . . . . . 117

[46] General representation theory of Jordan algebras . . . . . . . . . . 127

[47] Une généralisation du théorème d'Engel ................... 149

[48] Homomorphisms of Jordan rings of self-adjoint elements

(with C. E. Rickart) . . . . . . . . . . . . . . . . . . . . . . . . 152

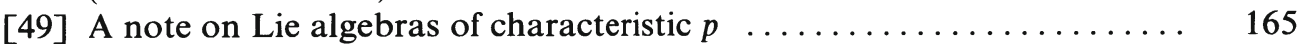

[50] Operator commutativity in Jordan algebras ................. 169

[51] A Kronecker factorization theorem for Cayley algebras and the exceptional simple Jordan algebra ....................... 173

[52] Structure of alternative and Jordan bimodules .............. 179

[53] A note on automorphisms and derivations of Lie algebras ........ 251

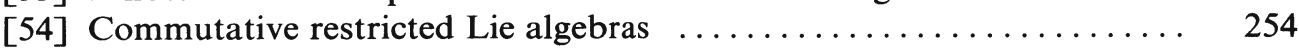

[55] A note on two dimensional division ring extensions $\ldots \ldots \ldots \ldots \ldots \ldots 261$

Personal History and Commentary $(1956-1965) \ldots \ldots \ldots \ldots \ldots \ldots . \ldots \ldots$

Research Papers, 1956-1965

[56] A theorem on the structure of Jordan algebras . . . . . . . . . . 290

[57] Generation of separable and central simple algebras ............. 299

[58] On reduced exceptional simple Jordan algebras

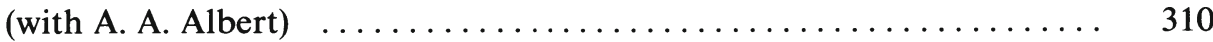

[59] On Jordan algebras with two generators (with L. J. Paige) . . . . . . 329

[60] Composition algebras and their automorphisms ............. 341

[61] Nilpotent elements in semi-simple Jordan algebras . . . . . . . . . 367

[62] A note on three dimensional simple Lie algebras . . . . . . . . . . . . 379

[63] Some groups of transformations defined by Jordan algebras I . . . . . 388

[64] Some groups of transformations defined by Jordan algebras II ..... 406

[65] Some groups of transformations defined by Jordan algebras III ..... 431

[66] MacDonald's theorem on Jordan algebras .................. 457

[67] A coordinatization theorem for Jordan algebras ............ 468

[68] A note on automorphisms of Lie algebras ................ 475 
[69] Generic norm of an algebra

489

[70] Clifford algebras for algebras with involution of type $D \ldots \ldots \ldots \ldots \ldots, 516$

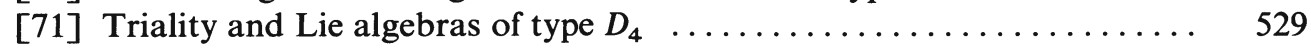

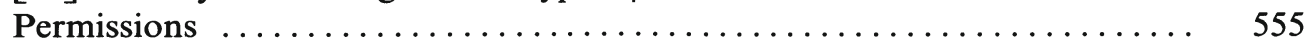

